\title{
MA Defence: "Shaping The Genohype: A Cautionary Tale of Overpromise" by Stephan Struve
}

\author{
Vu Thuy Anh Phan \\ School of Communication \\ Simon Fraser University \\ Our dear colleague, Stephan Struve, will defend his MA thesis on Thursday, December $3^{\text {rd }}$, 2015 at \\ Harbour Centre, Room 2200 (10:00 am -12:00 pm). Here is the abstract of his thesis "Shaping the \\ Genohype: A Cautionary Tale of Overpromise".
}

\begin{abstract}
This thesis examines the mechanisms at play in the hype of genomic science. While media are the primary conduit for scientific information, scientists and scholars claim that a variety of social forces shape this genohype. This in turn is driving unrealistic expectations about the potential application of genomic discoveries. This study will add qualitative empirical evidence about these social forces by examining the scientific process itself, as well as the role of the media and the public opinion. I conducted 12 in-depth, semi-structured interviews with genomic researchers, scientists and clinicians and used thematic analysis to explore how various social forces are shaping scientific work and the genohype. This study discovered evidence of a third-person effect and highlights how PR departments of universities and research facilities play an important role in 'pitching' genomic science to the media. Understanding these mechanisms at play can help manage expectations about the potential application of genomic discoveries. This research will ultimately benefit the media, scientists, decision makers, and members of the public by increasing knowledge and decreasing communicative barriers.
\end{abstract}

Keywords: Genohype; genomics; hype; hyperbole; overpromise; science communication 